\title{
A Completely Discrete Heterogeneous Multiscale Finite Element Method for Multiscale Richards' Equation of van Genuchten-Mualem Model
}

\author{
Haitao Cao, ${ }^{1,2}$ Tao Yu, ${ }^{3}$ and Xingye Yue ${ }^{2}$ \\ ${ }^{1}$ Department of Mathematics and Physics, Hohai University, Changzhou Campus, Changzhou 213022, China \\ ${ }^{2}$ Department of Mathematics, Soochow University, Suzhou, Jiangsu 215006, China \\ ${ }^{3}$ Department of Mathematics, Jinggangshan University, Jian, Jiangxi 343009, China \\ Correspondence should be addressed to Tao Yu; 20104007004@suda.edu.cn
}

Received 21 October 2013; Revised 24 January 2014; Accepted 11 February 2014; Published 20 March 2014

Academic Editor: Roberto Natalini

Copyright (C) 2014 Haitao Cao et al. This is an open access article distributed under the Creative Commons Attribution License, which permits unrestricted use, distribution, and reproduction in any medium, provided the original work is properly cited.

\begin{abstract}
We propose a fully discrete method for the multiscale Richards' equation of van Genuchten-Mualem model which describes the flow transport in unsaturated heterogenous porous media. Under the framework of heterogeneous multiscale method (HMM), a fully discrete scheme combined with a regularized procedure is proposed. Including the numerical integration, the discretization is given by $C^{0}$ piecewise finite element in space and an implicit scheme in time. Error estimates between the numerical solution and the solution of homogenized problem are derived under the assumption that the permeability is periodic. Numerical experiments with periodic and random permeability are carried out for the van Genuchten-Mualem model of Richards' equation to show the efficiency and accuracy of the proposed method.
\end{abstract}

\section{Physical Model}

Richards' equation is most often used to model the movement of groundwater flow in saturated-unsaturated porous media. It was formulated by Richards in 1931 [1]. It is a nonlinear partial differential parabolic equation. Depending on the saturation and pressure, three main forms of Richards' equation are usually presented: pressure-based form, saturation-based form, or mixed form. Combining the continuity equation with Darcy's law, the mixed form can be expressed as

$$
\partial_{t} \Theta(p)-\nabla\left(K\left(\frac{x}{\epsilon}, \Theta(p)\right) \nabla(p+z)\right)=0,
$$

where the reduced saturation is defined by $\Theta=\left(\theta-\theta_{r}\right) /\left(\theta_{s}-\right.$ $\left.\theta_{r}\right) \in[0,1] . \theta_{r}$ (the residual fluid content) and $\theta_{s}$ (the fluid content at saturation) are constants relying on the porous medium. $\theta$ denotes the saturation and $p$ denotes the pressure. Due to the complex heterogeneity of natural medium, the permeability $K(x / \epsilon, \Theta(p))$ of the medium oscillates rapidly with large contrast. $\epsilon(>0)$ is the characteristic length presenting the small scale variability of the media. The coordinate in the direction of gravity is denoted by $z$.

In this paper, we adopt the retention curves proposed by van Genuchten [2] and Mualem [3]. The relations of the reduced saturation $\Theta$, pressure $p$, and permeability $K$ are read as

$$
\begin{aligned}
\Theta(p) & = \begin{cases}\frac{1}{\left(1+|c p|^{n}\right)^{m}} & \text { for } p \leq 0, \\
1 & \text { for } p>0,\end{cases} \\
K\left(\frac{x}{\epsilon}, \Theta\right) & =K_{s}\left(\frac{x}{\epsilon}\right) K_{r}(\Theta) \\
& =K_{s}\left(\frac{x}{\epsilon}\right) \cdot \Theta^{1 / 2}\left[1-\left(1-\Theta^{1 / m}\right)^{m}\right]^{2},
\end{aligned}
$$

where $c, m \in(0,1)$ and $n=1 /(1-m)$ are the parameters of porous medium. $K_{s}$ and $K_{r}$ can be presented as the absolute and relative permeability, respectively. When $p>0, \Theta=1$, 
the medium is in the saturated state; otherwise, it is in the unsaturated case.

Under the unsaturated case, Richards' equation can also be rewritten in terms of reduced saturation by using the above relations. Consider

$$
\partial_{t} \Theta-\nabla\left(D\left(\frac{x}{\epsilon}, \Theta\right) \nabla \Theta+\overrightarrow{K_{z}}\left(\frac{x}{\epsilon}, \Theta\right)\right)=0,
$$

with the moisture diffusivity $D$ defined by

$$
\begin{aligned}
D\left(\frac{x}{\epsilon}, \Theta\right)= & -K\left(\frac{x}{\epsilon}, \Theta\right) \frac{\partial p}{\partial \Theta} \\
= & \frac{(1-m) K_{s}(x / \epsilon)}{\alpha m\left(\theta_{s}-\theta_{r}\right)} \Theta^{(1 / 2)-(1 / m)} \\
& \times\left[\left(1-\Theta^{1 / m}\right)^{m}+\left(1-\Theta^{1 / m}\right)^{-m}-2\right] \\
\doteq & D_{s}\left(\frac{x}{\epsilon}\right) D_{r}(\Theta) .
\end{aligned}
$$

Here, under the relations (2), we should point out that (1) can model both saturated and unsaturated cases. In saturated region, (1) is an elliptic equation; in unsaturated region, the equation is parabolic. On the other hand, in unsaturated case, (1) can be degenerated because $K_{r}$ may approximate to zero when $\Theta \rightarrow 0$ (in almost completely dry region). Richards' equation (3) can only model the unsaturated case. But (3) may also be degenerated, because the diffusivity $D$ may vanish or explode (see Figure 1(a)). For $\Theta=0$ (in almost completely dry region), the diffusivity $D_{r}$ vanishes, while for $\Theta$ going to 1 (in almost full saturated area) $D_{r}$ goes to infinity. The degeneracy of (1) and (3) leads to the fact that the solutions must be understood in the sense of distribution as proposed in [4-7].

Numerous papers have been published on numerical scheme for the non-multiscale degenerate parabolic problem (including Richards' equation). For example, in [5-9], the authors firstly regularized the degenerated problem then used the FEM or mixed FEM for spatial discretization. In [10-13], considering the time discretization aspect, the authors developed some schemes for time discretization, such as linear time discretization, adaptive time stepping, or relaxation scheme. The relaxation-iteration scheme analyzed in [13] for the fast diffusion case is a fixed point iteration which was proposed in [8] (also see [12] for the mixed finite element context).

Here, we should point out the work of $[6,7]$. In [6], the authors considered a class of degenerate parabolic equations including Stefan problem, porous-medium problem, and nonstationary filtration problem. Combining with a regularization approach (when necessary), the authors developed their fully discrete scheme including FEM in spatial and Backward-Euler semi-implicit scheme in time. At the same time, the effects of numerical integration and domain change were taken into account. In [7], under the relations (2), Backward-Euler implicit scheme in time with a regularization step for non-multiscale Richards' equation (3) was established and analyzed.
On the other hand, for this kind of multiscale problem, it is impossible to account explicitly for the spatial variability at fine scale because of the computational resource limitations in realistic situation. So, traditional numerical methods such as standard finite element methods (FEM) and finite difference methods (FDM) are generally not capable of solving this multiscale problem directly. In recent years, a number of multiscale numerical methods, such as multiscale finite element method (MsFEM) [14], heterogeneous multiscale method (HMM) [15], and upscaling method [16], have been proposed to solve the general multiscale problems based on similar ideas. Among them, the so-called heterogeneous multiscale method (HMM) has proved to be an efficient tool to assemble information from microscale problems in order to perform macroscale simulations. In [17], the author discussed the modeling and analysis of finite element methods (such as hybrid methods, coupling spectral or discontinuous Galerkin methods with FEM) for multiscale problems constructed in the framework of the HMM for multiscale PDEs (such as elliptic and parabolic equations).

To our knowledge, there are few papers about the numerical analysis of multiscale Richards' equation of van Genuchten-Mualem model. Based on the frame of HMMFEM, we will use the idea of $[6,7]$ to develop a fully discrete method for multiscale Richards' equation (5). In order to overcome the above difficulties, we regularize the equation firstly; secondly, a fully discrete multiscale numerical method based on heterogeneous multiscale method (HMM) [15] and FEM is developed on a macroscale mesh; at the same time, we use the numerical quadrature for computing the integral over each element. In the paper, we are only interested in (3).

Noticing that $D(x / \epsilon, \Theta)$ is separable, we set $\psi(\Theta)=$ $\int_{0}^{\Theta} D_{r}(s) d s$ (see Figure 1(b)). By Kirchhoff transform [4], we rewrite (3) as

$$
\partial_{t} \Theta-\nabla\left(D_{s}\left(\frac{x}{\epsilon}\right) \nabla \psi(\Theta)+\overrightarrow{K_{z}}\left(\frac{x}{\epsilon}, \Theta\right)\right)=0
$$

with $\psi^{\prime}(\Theta)=D_{r}(\Theta)$. So, when $\psi^{\prime}(\Theta) \rightarrow 0(\Theta \rightarrow 0)$, (5) may degenerate to hyperbolic equation; on the other hand, $\psi^{\prime}(\Theta) \rightarrow+\infty(\Theta \rightarrow 1)$, and (5) may transfer to elliptic equation.

Notations. $\Omega \subset R^{d}(d \geq 1)$ is a bounded polyhedral domain with boundary $\partial \Omega$. Set $Q=\Omega \times[0, T]$, where $T>0$ is fixed. For defining a solution in weak sense, we let $(\cdot, \cdot)$ stand for the inner product on $L^{2}(\Omega)$ or the duality pairing between $H^{-1}(\Omega)$ and $H_{0}^{1}(\Omega),\|\cdot\|_{0}$ stand for the norm in $L^{2}(\Omega)$, and $\|\cdot\|_{-1}$ and $\|\cdot\|_{1}$ stand for the norm in $H^{-1}(\Omega)$ and $H_{0}^{1}(\Omega)$.

\section{Formulation of the Problem}

In this paper, we will consider the following general nonlinear parabolic equation which includes Richards' equation (5):

$$
\begin{gathered}
\partial_{t} u^{\epsilon}-\nabla\left(a\left(\frac{x}{\epsilon}\right) \nabla \beta\left(u^{\epsilon}\right)+g\left(\frac{x}{\epsilon}, u^{\epsilon}\right)\right)=0, \quad \text { in } Q, \\
u^{\epsilon}(t=0)=u_{0}(x), \quad \text { in } \Omega, \\
u^{\epsilon}=0, \quad \text { on } \partial \Omega \times(0, T] .
\end{gathered}
$$




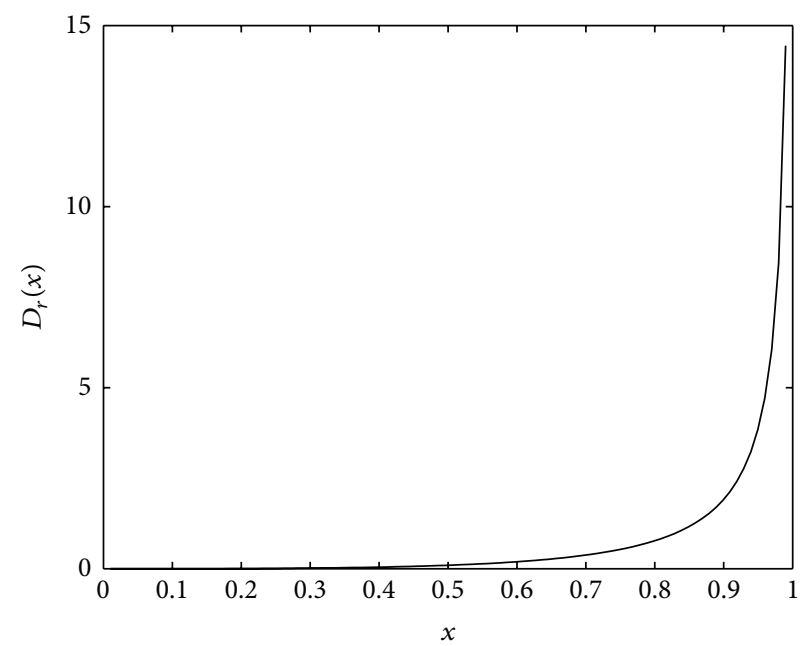

(a)

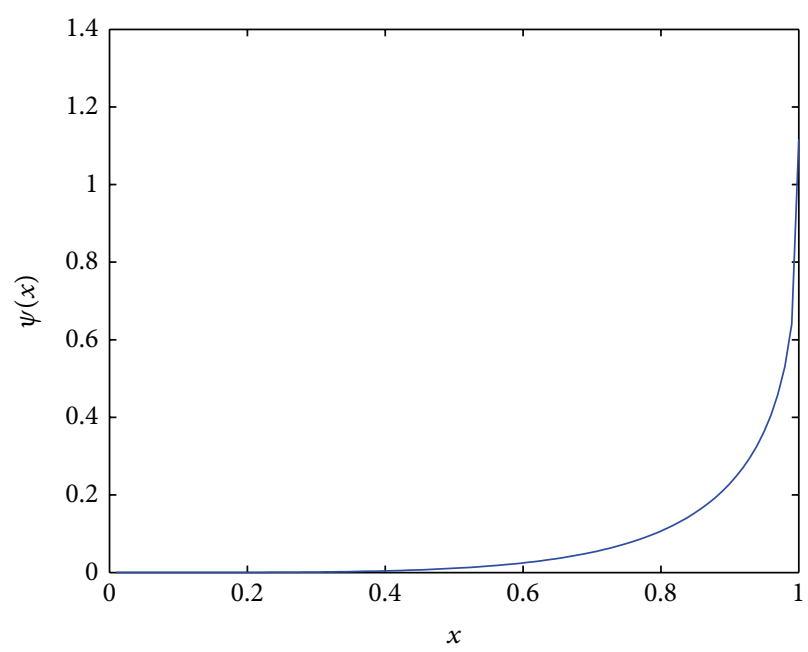

(b)

Figure 1: (a) $\operatorname{Dr}(x)$ function. (b) $\psi(x)$ function.

Assumption 1. (H1) $\beta$ is a maximal monotone graph in $[0,1] \times$ $R$ and $\beta(0)=0$.

(H2) For all $s \in(0,1], \beta^{\prime}(s)>0$ and $\beta^{\prime}(s)$ has the following asymptotic behavior:

$$
\begin{aligned}
\exists \alpha>0, \quad 0<\gamma<1, & \\
\text { s.t. } & \beta^{\prime}(s) \sim O\left(s^{\alpha}\right)(s \longrightarrow 0), \\
& \beta^{\prime}(s) \sim O\left((1-s)^{-\gamma}\right)(s \longrightarrow 1) .
\end{aligned}
$$

(H3) $0 \leq u_{0}(x) \leq 1$ almost everywhere.

(H4) The matrix $a(x / \epsilon)=\left(a_{i j}(x / \epsilon)\right)_{i, j=1}^{d}$ is bounded and uniformly elliptic. There exist constants $C_{a}$ and $C_{\bar{a}}$ such that $C_{a}\|\xi\|^{2} \leq(a(y) \xi, \xi) \leq C_{\bar{a}}\|\xi\|^{2}, \forall \xi \in R^{d}$.

(H5) $g(y, s): R^{n} \times R \mapsto R^{n}$ is bounded, uniformly Lipschitz continuous and satisfying

$$
\left|g\left(\cdot, u_{1}\right)-g\left(\cdot, u_{2}\right)\right|^{2} \leq C\left(u_{1}-u_{2}\right)\left(\beta\left(u_{1}\right)-\beta\left(u_{2}\right)\right) .
$$

A weak solution of problem (6) is defined as follows.

Definition 2. Find $u^{\epsilon} \in H^{1}\left(0, T ; H^{-1}(\Omega)\right)$ such that $u^{\epsilon}(0)=$ $u_{0}$ and $v^{\epsilon}=\beta\left(u^{\epsilon}\right) \in L^{2}\left(0, T ; H_{0}^{1}(\Omega)\right)$ and, $\forall \varphi \quad \epsilon$ $L^{2}\left(0, T ; H_{0}^{1}(\Omega)\right)$,

$$
\begin{gathered}
\left(\partial_{t} u^{\epsilon}, \varphi\right)_{\mathrm{Q}}+\left(a^{\epsilon}\left(\frac{x}{\epsilon}, u^{\epsilon}\right) \nabla \beta\left(u^{\epsilon}\right), \nabla \varphi\right)_{\mathrm{Q}} \\
+\left(g^{\epsilon}\left(\frac{x}{\epsilon}, u^{\epsilon}\right), \nabla \varphi\right)_{\mathrm{Q}}=0 .
\end{gathered}
$$

Remark 3. The existence, uniqueness, and regularity results for the above problem can be found in [4]. The solution of problem (6) may denote the reduced saturation and therefore should be bounded by 0 and 1 . In the setting stated above, the maximum principle holds for problem (6), so the solution remains bounded by 0 and 1 .
Remark 4. According to the constitution relations of the van Genuchten-Mualem, the parameter in (H2) is determined as $\alpha=(1 / 2)+(1 / m)$ and $\gamma=m(m \in(0,1))$. In Richards' equation, the convective function $g(y, u)$ denotes the permeability $K_{s}(y) K_{r}(u) \vec{e}_{z}$. And it is easy to verify that $\left(K_{r}\right)^{\prime}(u) \sim O\left(u^{(2 / m)-(1 / 2)}\right)$ and $\beta^{\prime}(u) \sim O\left(u^{(1 / m)+(1 / 2)}\right)$ when $u$ approaches to zero. Also, it is easy to verify that condition (8) is valid when the second variable approaches to zero. The other cases are obvious.

Let $0<\delta<1$ be a small perturbed parameter. We approximate $\beta$ by, for all $s \in[0,1]$,

$$
\beta_{\delta}(s)=\int_{0}^{s} \max \left\{\delta, \min \left\{\frac{1}{\delta}, \beta^{\prime}(s)\right\}\right\} d s .
$$

So, we get

$$
\beta_{\delta}^{\prime}(s)= \begin{cases}\delta, & \text { if } 0 \leq s \leq C \delta^{1 / \alpha}, \\ \beta^{\prime}(s), & \text { otherwise, } \\ \frac{1}{\delta}, & \text { if } 1-C \delta^{1 / \gamma} \leq s \leq 1 .\end{cases}
$$

Hence, $\beta_{\delta}^{-1}$ is also a maximal monotone graph in $R \times[0,1]$ and it follows that

$$
\begin{gathered}
\delta \leq\left(\beta_{\delta}^{-1}\right)^{\prime}(x) \leq \frac{1}{\delta} \\
\left|\beta_{\delta}^{-1}(x)-\beta^{-1}(x)\right| \leq C \delta^{\mu}, \quad \mu=\min \left\{\frac{1}{\gamma}, \frac{1}{\alpha}\right\} .
\end{gathered}
$$

Let $T_{H}=\{K\}_{H}$ be a regular triangle decomposition of $\Omega$, where $H$ stands for the mesh size. Define the functional space

$$
X_{H}=\left\{\chi \text { is linear for all } K \in T_{H}\right\},
$$

$X_{H}^{0}=\left\{\chi\right.$ is linear for all $K \in T_{H} ; \chi=0$ on $\left.\partial \Omega\right\}$. 
In order to simplify the computation, we will use the numerical integration scheme as in [6]. Let $\Pi_{H}$ be the local linear interpolant operator; then,

$$
(\phi, \varphi)_{H, K} \doteq \int_{K} \Pi_{H}(\phi \varphi) d x,
$$

for any piecewise uniformly continuous functions $\phi$ and $\varphi$. So, for all $W, V \in X_{H}$ and every element $K$, the numerical integration scheme satisfies that [6]

$$
E(W, V) \doteq\left|(W, V)_{K}-(W, V)_{H, K}\right| \leq C H\|W\|_{L^{2}(K)}\|V\|_{H^{1}(K)},
$$

$E(W, V) \doteq\left|(W, V)_{K}-(W, V)_{H, K}\right| \leq C H^{2}\|W\|_{H^{1}(K)}\|V\|_{H^{1}(K)}$,

$$
\begin{gathered}
C_{1}\|W\|_{L^{2}(K)}^{2} \leq(W, W)_{H, K} \leq C_{2}\|W\|_{L^{2}(K)}^{2}, \\
\left|(W, V)_{H, K}\right| \leq C_{2}\|W\|_{L^{2}(K)}\|V\|_{L^{2}(K)}
\end{gathered}
$$

and, for any $b(x) \in W^{1, \infty}(K)[18]$,

$$
E(b W, V) \leq C H\|b\|_{W^{1, \infty}(K)}\|W\|_{H^{1}(K)}\|V\|_{H^{1}(K)} .
$$

$I_{H}$ stands for the piecewise linear interpolant operator and satisfies

$$
\left\|I_{H} \varphi-\varphi\right\|_{L^{2}(\Omega)} \leq C H\|\nabla \varphi\|_{L^{2}(\Omega)}, \quad \varphi \in H^{1}(\Omega),
$$

and $E_{H}$ stands for the discrete $L^{2}$ projection operator; more precisely, for any $z \in L^{2}(\Omega), E_{H} z \in X_{H}$ and satisfies

$$
\left\|E_{H} z-z\right\|_{H^{-1}(\Omega)} \leq C H\|z\|_{L^{2}(\Omega)} .
$$

Let $x_{r}$ and $\omega_{K, r}(r=\overline{1, L})$ denote the quadrature nodes and weights in $K$. Let $N$ be an integer and let $\tau=T / N$. Our fully discrete formulation for problem (6) is read as follows: find $v^{i, H} \in X_{H}^{0}, u^{i, H}=I_{H} \beta_{\delta}^{-1}\left(v^{i, H}\right) \in X_{H}(i=\overline{1, N})$, such that

$$
\begin{aligned}
& \sum_{K}\left(\frac{u^{i, H}-u^{i-1, H}}{\tau}, \chi\right)_{H, K}+\sum_{K} A_{H}\left(v^{i, H}, \chi\right) \\
& \quad+\sum_{K} G_{H}\left(u^{i, H}, \chi\right)=0, \quad \forall \chi \in X_{H}^{0}, \\
& v^{0, H}=I_{H} \beta\left(u^{0}\right), u^{0, H}=E_{H} u^{0},
\end{aligned}
$$

where

$$
\begin{aligned}
& A_{H}\left(v^{i, H}, \chi\right) \\
& \quad \doteq \sum_{r=1}^{L} \frac{\omega_{K, r}}{\left|I_{l}\left(x_{r}\right)\right|} \int_{I_{l}\left(x_{r}\right)} a\left(\frac{x}{\epsilon}\right) \nabla R_{1}\left(v^{i, H}\right) \nabla R_{1}(\chi) d x \\
& \quad \doteq\left(\widehat{a} \nabla v^{i, H}, \nabla \chi\right)_{H, K^{\prime}} \\
& G_{H}\left(u^{i, H}, \chi\right) \\
& \quad \doteq \sum_{r=1}^{L} \frac{\omega_{K, r}\left|I_{l}\left(x_{r}\right)\right|}{\int_{I_{l}\left(x_{r}\right)}} g\left(\frac{x}{\epsilon}, u^{i, H}\right) \nabla R_{1}(\chi) d x \\
& \quad \doteq\left(\widehat{g}\left(u^{i, H}\right), \nabla \chi\right)_{H, K^{\prime}}
\end{aligned}
$$

and the operator $R_{1}$ is defined by the following problem:

$$
\begin{gathered}
-\nabla\left(a^{\epsilon}(x) \nabla R_{1}(\chi)\right)=0 \quad \text { in } I_{l}\left(x_{r}\right), \\
R_{1}(\chi)=\chi_{r} \quad \text { on } \partial I_{l}\left(x_{r}\right),
\end{gathered}
$$

where $\chi_{r}=\chi\left(x_{r}\right)+\chi^{\prime}\left(x_{r}\right)\left(x-x_{r}\right)$ and the cell $I_{l}\left(x_{r}\right)$ is a square of size $l$ centered at $x_{r}$. So far, our fully discrete HMMFEM is settled down and the porous media do not have to be periodic.

\section{Some Results on the Homogenized Problem}

In this section, we will review the results of homogenization and the analysis of the discrete homogenized problem. Under the assumptions (H1)-(H5) and if the function $a(y)$ and $g(y, s)$ are periodic in $y$ with unit square $Y$. In [19], the authors have established the homogenization theory for (6). It has been shown that, in suitable topology space, the solutions $u^{\epsilon}$ of problem (6) converge to the solution of the following problem as $\epsilon \rightarrow 0$.

$$
\begin{gathered}
\partial_{t} u-\nabla\left(a^{*} \nabla \beta(u)+g^{*}(u)\right)=0, \quad \text { in } Q, \\
u(t=0)=u_{0}(x), \quad \text { in } \Omega, \\
u=0, \quad \text { on } \partial \Omega \times(0, T],
\end{gathered}
$$

where $a^{*}=\left(a_{i j}^{*}\right)_{1 \leq i, j \leq d}$ and $g^{*}=\left(g_{i}^{*}\right)_{i=1}^{d}$ are defined as

$$
\begin{aligned}
& \left(a^{*}\right)_{i j}=\int_{Y}\left(a_{i j}(y)+a_{i k}(y) \frac{\partial \Lambda^{k}(y)}{\partial y_{j}}\right) d y, \\
& g^{*}(s)=\int_{Y}\left(g(y, s)+a(y) \nabla_{y} \eta(y, s)\right) d y,
\end{aligned}
$$

and $\Lambda^{k}$ and $\eta$ are the periodic solutions of following cell problems, respectively.

$$
\begin{gathered}
-\nabla_{y} \cdot\left(a(y) \nabla_{y} \Lambda^{k}(y)\right)=\nabla_{y} \cdot\left(a(y) \cdot e_{k}\right), \quad \text { in } Y, \\
\int_{Y} \Lambda^{k} d y=0, \\
-\nabla_{y} \cdot\left(a(y) \nabla_{y} \eta(y, s)\right)=\nabla_{y} \cdot(g(y, s)), \quad \text { in } Y, \\
\int_{Y} \eta d y=0,
\end{gathered}
$$

and $a^{*}$ and $g^{*}$ inherit the property of $a$ and $g$ [16].

Remark 5. According to Alt and Luckhaus [4], we have at least $u \in L^{\infty}\left(0, T ; L^{1}(\Omega)\right)$ and $u_{t} \in L^{2}\left(0, T ; H^{-1}(\Omega)\right)$. Moreover, the maximum principle leads to $u \in L^{\infty}\left(0, T ; L^{\infty}(\Omega)\right)$. We therefore conclude that $u \in C\left(0, T ; L^{2}(\Omega)\right)$. This gives us $u(t, \cdot)$ pointwise for every $t \in[0, T]$. 
The problem (26) can be read as follows.

Definition 6. For every time interval $\left[t_{i-1}, t_{i}\right] \subset[0, T]$, find $u\left(t_{i}\right) \in L^{2}(\Omega)$ such that $\int_{t_{i-1}}^{t_{i}} \beta(u(t)) d t \in H_{0}^{1}(\Omega)$ and, for all $\varphi \in H_{0}^{1}(\Omega)$,

$$
\begin{gathered}
\left(u\left(t_{i}\right)-u\left(t_{i-1}\right), \varphi\right)_{\Omega}+\left(\int_{t_{i-1}}^{t_{i}} a^{*} \nabla \beta(u) d t, \nabla \varphi\right)_{\Omega} \\
+\left(\int_{t_{i-1}}^{t_{i}} g^{*}(u) d t, \nabla \varphi\right)_{\Omega}=0 .
\end{gathered}
$$

We also give out the fully discrete scheme for the problem (26) by a regularization procedure.

Definition 7. Find $V^{i} \in X_{H}^{0}$ such that $U^{i}=I_{H} \beta_{\delta}^{-1}\left(V^{i}\right) \in X_{H}$ and, for all $W \in X_{H}^{0}$,

$$
\begin{gathered}
\left(U^{i}-U^{i-1}, W\right)_{H}+\tau\left(a^{*} \nabla V^{i}, \nabla W\right)_{H} \\
+\tau\left(I_{H} g^{*}\left(U^{i}\right), \nabla W\right)_{H}=0, \\
V^{0}=I_{H} \beta\left(u^{0}\right), \quad U^{0}=E_{H} u^{0},
\end{gathered}
$$

where $(U, W)_{H}=\sum_{K \in T_{H}}(U, W)_{H, K}$.

In the rest of this section, we will give out a prior estimate for problem (31) and the error estimate of $V^{i}-\beta\left(u\left(t_{i}\right)\right)$ and $U^{i}-u\left(t_{i}\right)$.

Theorem 8. Under the assumptions (H1) to (H5), there exist a positive constant $C$ independent of $H, \delta, \epsilon$ such that

$$
\begin{gathered}
\max _{1 \leq i \leq N}\left\|U^{i}\right\|_{L^{\infty}(\Omega)}+\delta \max _{1 \leq i \leq N}\left\|V^{i}\right\|_{L^{2}(\Omega)}^{2}+\sum_{i=1}^{N} \tau\left\|V^{i}\right\|_{H^{1}(\Omega)}^{2} \leq C, \\
\sum_{i=1}^{N} \delta\left(\left\|V^{i}-V^{i-1}\right\|_{0}^{2}+\left\|U^{i}-U^{i-1}\right\|_{0}^{2}\right) \leq C .
\end{gathered}
$$

Proof. Let $W=V^{i}$ in (31) and sum it over $i$ from 1 to $N$.

$$
\begin{gathered}
\sum_{i=1}^{N}\left(U^{i}-U^{i-1}, V^{i}\right)_{H}+\sum_{i=1}^{N} \tau\left(a^{*} \nabla V^{i}, \nabla V^{i}\right)_{H} \\
+\sum_{i=1}^{N} \tau\left(I_{H} g^{*}\left(U^{i}\right), \nabla V^{i}\right)_{H}=0 .
\end{gathered}
$$

We will estimate each term separately. Following the idea in [6], we have

$$
\begin{aligned}
& \sum_{i=1}^{N}\left(U^{i}-U^{i-1}, V^{i}\right)_{H} \\
& \quad=\sum_{i=1}^{N}\left(I_{H} \beta_{\delta}^{-1}\left(V^{i}\right)-I_{H} \beta_{\delta}^{-1}\left(V^{i-1}\right), V^{i}\right)_{H} \\
& \quad \geq \delta\left\|V^{N}\right\|_{0}^{2}-C .
\end{aligned}
$$

Using (H4), (H5) and Poincaré inequality, we have

$$
\begin{aligned}
& \sum_{i=1}^{N} \tau\left(a^{*} \nabla V^{i}, \nabla V^{i}\right)_{H} \geq C \sum_{i=1}^{N} \tau\left\|\nabla V^{i}\right\|_{0}^{2} \geq C \sum_{i=1}^{N} \tau\left\|V^{i}\right\|_{1}^{2}, \\
& \left|\sum_{i=1}^{N} \tau\left(I_{H} g^{*}\left(U^{i}\right), \nabla V^{i}\right)_{H}\right| \leq \sum_{i=1}^{N} \tau\left\|I_{H} g^{*}\left(U^{i}\right)\right\|_{0}\left\|\nabla V^{i}\right\|_{0} \\
& \leq C+\eta \sum_{i=1}^{N} \tau\left\|\nabla V^{i}\right\|_{0}^{2}
\end{aligned}
$$

Since $U_{i}=I_{H} \beta_{\delta}^{-1}\left(V^{i}\right)$, we have $\max _{1 \leq i \leq N}\left\|U^{i}\right\|_{L^{\infty}(\Omega)} \leq$ $C$. Combining all the terms and choosing $\eta$ properly, we complete the first part.

Again, we let $W=\left(V^{i}-V^{i-1}\right)$ in (31) and sum it over $i$ from 1 to $N$.

$$
\begin{gathered}
\sum_{i=1}^{N}\left(U^{i}-U^{i-1}, V^{i}-V^{i-1}\right)_{H}+\sum_{i=1}^{N} \tau\left(a^{*} \nabla V^{i}, \nabla\left(V^{i}-V^{i-1}\right)\right)_{H} \\
+\sum_{i=1}^{N} \tau\left(I_{H} g^{*}\left(U^{i}\right), \nabla\left(V^{i}-V^{i-1}\right)\right)_{H}=0 .
\end{gathered}
$$

Considering that $V^{i}=I_{H} \beta_{\delta}^{-1}\left(U^{i}\right)$, we have

$$
\begin{aligned}
& \sum_{i=1}^{N}\left(U^{i}-U^{i-1}, V^{i}-V^{i-1}\right)_{H} \\
& \quad \geq C \sum_{i=1}^{N} \delta\left(\left\|U^{i}-U^{i-1}\right\|_{0}^{2}+\left\|V^{i}-V^{i-1}\right\|_{0}^{2}\right) .
\end{aligned}
$$

By (H4), (H5) and (32), it is easy to get

$$
\begin{aligned}
& \mid \sum_{i=1}^{N} \tau\left(a^{*} \nabla V^{i}, \nabla\left(V^{i}-V^{i-1}\right)\right)_{H} \\
& \quad+\sum_{i=1}^{N} \tau\left(I_{H} g^{*}\left(U^{i}\right), \nabla\left(V^{i}-V^{i-1}\right)\right)_{H} \mid \leq C .
\end{aligned}
$$

So, we finish the second part of the theorem.

Define a regular G-operator $H^{-1} \rightarrow H_{0}^{1}$ and $G_{H^{-}}$ operator $H^{-1} \rightarrow X_{H}^{0}$ as in [6], for all $\varphi \in H^{-1}$,

$$
\begin{gathered}
\left(a^{*} \nabla G \varphi, \nabla \psi\right)=(\varphi, \psi), \quad \forall \psi \in H_{0}^{1}, \\
\left(a^{*} \nabla G \varphi, \nabla \chi\right)=\left(a^{*} \nabla G_{H} \varphi, \nabla \chi\right), \quad \forall \chi \in X_{H}^{0},
\end{gathered}
$$

and there is

$$
\left\|\left(G-G_{H}\right) \varphi\right\|_{H^{s}} \leq C H^{2-r-s}\|\varphi\|_{H^{-r}}, \quad 0 \leq r, s \leq 1 .
$$

Firstly, we have

$$
\begin{gathered}
\|\varphi\|_{-1}=\sup _{\psi \in H_{0}^{1}} \frac{|(\varphi, \psi)|}{\|\nabla \psi\|}, \\
C_{\underline{a}}\|\nabla G \varphi\|^{2} \leq\left(a^{*} \nabla G \varphi, \nabla G \varphi\right)=(\varphi, G \varphi) \leq\|\varphi\|_{-1}\|\nabla G \varphi\| .
\end{gathered}
$$


On the other hand,

$$
\|\varphi\|_{-1}=\sup _{\psi \in H_{0}^{1}} \frac{|(\varphi, \psi)|}{\|\nabla \psi\|}=\sup _{\psi \in H_{0}^{1}} \frac{\left|\left(a^{*} \nabla G \varphi, \nabla \psi\right)\right|}{\|\nabla \psi\|} \leq C_{\bar{a}}\|\nabla G \varphi\|,
$$

so we have $\|\varphi\|_{-1}$ equivalent to $\|\nabla G \varphi\|$. We also introduce the following two identities:

$$
\begin{aligned}
& \sum_{i=1}^{n}\left(a_{i}-a_{i-1}, b_{i}\right)=a_{n} b_{n}-a_{0} b_{0}-\sum_{i=1}^{n} a_{i-1}\left(b_{i}-b_{i-1}\right), \\
& \sum_{i=1}^{n}\left(a_{i}-a_{i-1}, a_{i}\right)=\left(a_{n}\right)^{2}-\left(a_{0}\right)^{2}+\sum_{i=1}^{n}\left(a_{i}-a_{i-1}\right)^{2} .
\end{aligned}
$$

Theorem 9. $u^{i}$ and $U^{i}$ are the solutions of problems (26) and (31) at time $t_{i}$, respectively. Denote that $\bar{u}^{i}=\left(\frac{1}{\tau}\right) \int_{I_{i}} u d t, e_{u}^{i}=$ $U^{i}-\bar{u}^{i}$, and $e_{v}^{i}=V^{i}-(1 / \tau) \int_{I_{i}} \beta(u) d t$. Under the assumptions (H1) to (H5), there exists a positive constant $C$ independent of $H, \delta, \epsilon, \tau$ such that

$$
\begin{aligned}
\max _{1 \leq i \leq N}\left\|e_{u}^{i}\right\|_{-1}^{2}+\sum_{i=1}^{N}\left\|e_{u}^{i}-e_{u}^{i-1}\right\|_{-1}^{2} \\
\quad+\sum_{i=1}^{N} \int_{I_{i}}\left(V^{i}-\beta(u), \beta^{-1}\left(V^{i}\right)-u(t)\right)_{\Omega} d t \\
\leq C\left(H+H^{2}+\delta^{2 \mu}+\delta^{\mu}+\tau+\frac{H}{\delta}+\frac{H^{4}}{\tau \delta^{2}}+\frac{H^{2}}{\delta^{2}}+\frac{H^{2}}{\sqrt{\tau \delta}}\right) .
\end{aligned}
$$

Remark 10. The proof method of Theorem 9 can be found in $[6,12]$. However, there is a little difference between our result and the result in $[6,12]$ because we consider the two degenerate points case.

\section{Main Result}

In this section, the main task is to give out the error between $V^{i}$ and $v^{i, H}$. Here, $V^{i}$ and $v^{i, H}$ are the solutions of problems (31) and (22), respectively. Before the proof of our main result, the following useful lemma will be introduced $[15,16]$.

Lemma 11. $a^{*}, g^{*}$ are defined in (27) and (28) and $\hat{a}, \hat{g}$ are defined in (23) and (24), respectively. Then, for all $s \in R$,

$$
\left|a^{*}(s)-\widehat{a}(s)\right| \leq C \frac{\epsilon}{l}, \quad\left|g^{*}(s)-\widehat{g}(s)\right| \leq C \frac{\epsilon}{l} .
$$

Then, we have the main result of the paper.

Theorem 12. $u^{i, H}, v^{i, H}$ and $U^{i}, V^{i}$ are the solutions of problems (22) and (31) at time $t_{i}$, respectively. Denote $e_{v, i}^{H}=v^{i, H}-V^{i} \in$
$X_{H}^{0}$ and $e_{u, i}^{H}=u^{i, H}-U^{i} \in X_{H}$. Then, under assumptions (H1)(H5), there exists a positive constant $C$ independent of $H, \delta, \epsilon$, $\tau$ such that

$$
\begin{aligned}
\max _{1 \leq i \leq N}\left\|e_{u, i}^{H}\right\|_{-1}^{2}+\sum_{i=1}^{N}\left\|e_{u, i}^{H}-e_{u, i-1}^{H}\right\|_{-1}^{2} \\
\quad+\tau \sum_{i=1}^{N}\left(v^{i, H}-V^{i}, \beta^{-1}\left(v^{i, H}\right)-\beta^{-1}\left(V^{i}\right)\right)_{\Omega} \\
\leq C\left(H+\frac{H}{\delta}+\frac{H^{2}}{\delta^{2}}+\delta^{\mu}+\delta^{2 \mu}+\frac{\epsilon^{2}}{l^{2}}\right) .
\end{aligned}
$$

Proof. Subtract (31) from (22) and notice the notation $\sum_{K}(\cdot, \cdot)_{H, K}=(\cdot, \cdot)_{H}$; we have

$$
\begin{aligned}
\left(u^{i, H}\right. & \left.-u^{i-1, H}-U^{i}+U^{i-1}, \chi\right)_{H} \\
& +\tau\left(A_{H}\left(v^{\epsilon, H}, \chi\right)-\left(a^{*} \nabla V^{i}, \nabla \chi\right)_{H}\right) \\
& +\tau\left(G_{H}\left(u^{\epsilon, H}, \chi\right)-\left(g^{*}\left(U^{i}\right), \nabla \chi\right)_{H}\right)=0 ;
\end{aligned}
$$

that is,

$$
\begin{aligned}
\left(u^{i, H}\right. & \left.-u^{i-1, H}-U^{i}+U^{i-1}, \chi\right)_{H} \\
& +\tau\left(\left(\widehat{a} \nabla v^{i, H}, \nabla \chi\right)_{H}-\left(a^{*} \nabla V^{i}, \nabla \chi\right)_{H}\right) \\
& +\tau\left(\left(\widehat{g}\left(u^{i, H}\right), \nabla \chi\right)_{H}-\left(g^{*}\left(U^{i}\right), \nabla \chi\right)_{H}\right)=0 .
\end{aligned}
$$

Let $\chi=G_{H} e_{u, i}^{H} \in X_{H}^{0}$ and sum $i=1 \cdots N$; then we have

$$
\begin{aligned}
& \sum_{i=1}^{N}\left(u^{i, H}-u^{i-1, H}-U^{i}+U^{i-1}, G_{H} e_{u, i}^{H}\right)_{H} \\
& +\tau \sum_{i=1}^{N}\left(\left(\widehat{a} \nabla v^{i, H}-a^{*} \nabla V^{i}, \nabla G_{H} e_{u, i}^{H}\right)_{H}\right) \\
& +\tau \sum_{i=1}^{N}\left(\left(\widehat{g}\left(u^{i, H}\right)-g^{*}\left(U^{i}\right), \nabla G_{H} e_{u, i}^{H}\right)_{H}\right)=0 .
\end{aligned}
$$

Denote the above equality by $T_{1}+T_{2}+T_{3}=0$.

For the term $T_{1}$, noticing that $a^{*}$ is also positive and bounded, we use (15), (45), and the definition of $G_{H}$ to get

$$
\begin{aligned}
T_{1}= & \sum_{i=1}^{N}\left(e_{u, i}^{H}-e_{u, i-1}^{H}, G_{H} e_{u, i}^{H}\right)_{H} \\
= & \sum_{i=1}^{N}\left(e_{u, i}^{H}-e_{u, i-1}^{H}, G_{H} e_{u, i}^{H}\right) \\
& +\sum_{i=1}^{N} \sum_{K} E\left(e_{u, i}^{H}-e_{u, i-1}^{H}, G_{H} e_{u, i}^{H}\right)
\end{aligned}
$$




$$
\begin{aligned}
\geq & \sum_{i=1}^{N}\left(a^{*} \nabla G_{H}\left(e_{u, i}^{H}-e_{u, i-1}^{H}\right), \nabla G_{H} e_{u, i}^{H}\right) \\
& -C H \sum_{i=1}^{N}\left\|e_{u, i}^{H}-e_{u, i-1}^{H}\right\|_{0}\left\|G_{H} e_{u, i}^{H}\right\|_{1} \\
\geq & \left(a^{*} \nabla G_{H} e_{u, N}^{H}, \nabla G_{H} e_{u, N}^{H}\right) \\
& +\sum_{i=1}^{N}\left(a^{*} \nabla G_{H}\left(e_{u, i}^{H}-e_{u, i-1}^{H}\right), \nabla G_{H}\left(e_{u, i}^{H}-e_{u, i-1}^{H}\right)\right) \\
& -\left(a^{*} \nabla G_{H} e_{u, 0}^{H}, \nabla G_{H} e_{u, 0}^{H}\right)-\eta \sum_{i=1}^{N}\left\|e_{u, i}^{H}-e_{u, i-1}^{H}\right\|_{-1}^{2} \\
& -C(\eta) H^{2} \sum_{i=1}^{N}\left\|e_{u, i}^{H}\right\|_{-1}^{2} \\
\geq & C_{\underline{a}}\left\|e_{u, N}^{H}\right\|_{-1}^{2}-C_{\bar{a}}\left\|e_{u, 0}^{H}\right\|_{-1}^{2} \\
& +\left(C_{\underline{a}}-\eta\right) \sum_{i=1}^{N}\left\|e_{u, i}^{H}-e_{u, i-1}^{H}\right\|_{-1}^{2}-C(\eta) H^{2} \sum_{i=1}^{N}\left\|e_{u, i}^{H}\right\|_{-1}^{2} .
\end{aligned}
$$

Rewrite the term $T_{2}$ as

$$
\begin{aligned}
T_{2}= & \tau \sum_{i=1}^{N}\left(a^{*} \nabla e_{v, i}^{H}, \nabla G_{H} e_{u, i}^{H}\right) \\
& +\tau \sum_{i=1}^{N} \sum_{K} E\left(a^{*} \nabla e_{v, i}^{H}, \nabla G_{H} e_{u, i}^{H}\right) \\
& +\tau \sum_{i=1}^{N}\left(\left(\widehat{a}-a^{*}\right) \nabla v^{i, H}, \nabla G_{H} e_{u, i}^{H}\right)_{H} \\
= & \tau \sum_{i=1}^{N}\left(e_{v, i}^{H}, e_{u, i}^{H}\right)+\tau \sum_{i=1}^{N} \sum_{K} E\left(a^{*} \nabla e_{v, i}^{H}, \nabla G_{H} e_{u, i}^{H}\right) \\
& +\tau \sum_{i=1}^{N}\left(\left(\widehat{a}-a^{*}\right) \nabla v^{i, H}, \nabla G_{H} e_{u, i}^{H}\right)_{H} \\
\doteq & T_{21}+T_{22}+T_{23} .
\end{aligned}
$$

Applying (20), (12), and (32), we get

$$
\begin{array}{r}
T_{21}=\tau \sum_{i=1}^{N}\left(v^{i, H}-V^{i}, \beta^{-1}\left(v^{i, H}\right)-\beta^{-1}\left(V^{i}\right)\right) \\
+\tau \sum_{i=1}^{N}\left(e_{v, i}^{H} I_{H} \beta_{\delta}^{-1}\left(v^{i, H}\right)-\beta^{-1}\left(v^{i, H}\right)\right. \\
\left.+I_{H} \beta_{\delta}^{-1}\left(V^{i}\right)-\beta^{-1}\left(V^{i}\right)\right) \\
\geq \tau \sum_{i=1}^{N}\left(v^{i, H}-V^{i}, \beta^{-1}\left(v^{i, H}\right)-\beta^{-1}\left(V^{i}\right)\right)
\end{array}
$$

$$
\begin{gathered}
-C \tau \sum_{i=1}^{N}\left\|e_{v, i}^{H}\right\|_{0}\left(H\left\|\nabla \beta_{\delta}^{-1}\left(v^{i, H}\right)\right\|\right. \\
\left.+H\left\|\nabla \beta_{\delta}^{-1}\left(V^{i}\right)\right\|+\delta^{\mu}\right) \\
\geq \tau \sum_{i=1}^{N}\left(v^{i, H}-V^{i}, \beta^{-1}\left(v^{i, H}\right)-\beta^{-1}\left(V^{i}\right)\right) \\
-C \tau \sum_{i=1}^{N}\left\|e_{v, i}^{H}\right\|_{0}\left(\frac{H}{\delta}\left\|\nabla v^{i, H}\right\|+\frac{H}{\delta}\left\|\nabla V^{i}\right\|+\delta^{\mu}\right) \\
\geq \tau \sum_{i=1}^{N}\left(v^{i, H}-V^{i}, \beta^{-1}\left(v^{i, H}\right)-\beta^{-1}\left(V^{i}\right)\right)-C\left(\frac{H}{\delta}+\delta^{\mu}\right), \\
\left|T_{22}\right| \leq C H \tau \sum_{i=1}^{N}\left\|a^{*}\right\|_{1, \infty}\left\|\nabla e_{v, i}^{H}\right\|_{0}\left\|^{-} \nabla G_{H} e_{u, i}^{H}\right\|_{0} \\
\leq C \sum_{i=1}^{N} \tau\left(H^{2}\left\|e_{v, i}^{H}\right\|_{1}^{2}+\left\|e_{u, i}^{H}\right\|_{1}^{2}\right) \\
\leq C\left(T_{23} \mid \leq C \frac{\epsilon}{l} \tau \sum_{i=1}^{N}\left\|\nabla v^{i, H}\right\| \sum_{i=1}^{N} \tau\left\|e_{u, i}^{H}\right\|_{-1} \leq C\left(\frac{\epsilon}{l}\right)^{H}\left\|_{1}^{2}\right\|_{1}^{2}+C \tau \sum_{i=1}^{N}\left\|e_{u, i}^{H}\right\|_{-1}^{2} .\right.
\end{gathered}
$$

For the terms $T_{22}, T_{23}$, we have used (19), (18), and Lemma 11. Similar to $T_{2}$, the term $T_{3}$ can be estimated as the following:

$$
\begin{aligned}
T_{3}= & \tau \sum_{i=1}^{N}\left(\hat{g}\left(u^{i, H}\right)-g^{*}\left(U^{i}\right), \nabla G_{H} e_{u, i}^{H}\right)_{H} \\
= & \tau \sum_{i=1}^{N}\left(\hat{g}\left(u^{i, H}\right)-g^{*}\left(u^{i, H}\right), \nabla G_{H} e_{u, i}^{H}\right)_{H} \\
& +\tau \sum_{i=1}^{N}\left(g^{*}\left(u^{i, H}\right)-g^{*}\left(U^{i}\right), \nabla G_{H} e_{u, i}^{H}\right)_{H} \\
\doteq & T_{31}+T_{32}, \\
\left|T_{31}\right| \leq & C \frac{{ }_{l}}{l} \tau \sum_{i=1}^{N}\left\|e_{u, i}^{H}\right\|_{-1} \leq C\left(\frac{\epsilon}{l}\right)^{2}+\tau \sum_{i=1}^{N}\left\|e_{u, i}^{H}\right\|_{-1}^{2}, \\
T_{32}= & \tau \sum_{i=1}^{N}\left(g^{*}\left(u^{i, H}\right)-g^{*}\left(U^{i}\right), \nabla G_{H} e_{u, i}^{H}\right) \\
& +\tau \sum_{i=1}^{N} E\left(g^{*}\left(u^{i, H}\right)-g^{*}\left(U^{i}\right), \nabla G_{H} e_{u, i}^{H}\right),
\end{aligned}
$$




$$
\begin{aligned}
\left|T_{32}\right| \leq \tau \sum_{i=1}^{N} & \left(\left\|g^{*}\left(u^{i, H}\right)-g^{*}\left(U^{i}\right)\right\|_{0}\right. \\
& \left.+C H\left(\left\|\nabla g^{*}\left(u^{i, H}\right)\right\|_{0}+\left\|\nabla g^{*}\left(U^{i}\right)\right\|_{0}\right)\right) \\
& \times\left\|e_{u, i}^{H}\right\|_{-1}
\end{aligned}
$$

Noticing $u^{i, H}=I_{H} \beta_{\delta}^{-1}\left(v^{i, H}\right)\left(\right.$ also $\left.U^{i}\right)$ and (H5), it follows that

$$
\begin{aligned}
\| g^{*}( & \left.u^{i, H}\right)-g^{*}\left(U^{i}\right) \|_{0}^{2} \\
\leq & \left\|g^{*}\left(\beta^{-1}\left(v^{i, H}\right)\right)-g^{*}\left(\beta^{-1}\left(V^{i}\right)\right)\right\|_{0}^{2} \\
& +\left\|g^{*}\left(u^{i, H}\right)-g^{*}\left(\beta^{-1}\left(v^{i, H}\right)\right)\right\|_{0}^{2} \\
& +\left\|g^{*}\left(\beta^{-1}\left(V^{i}\right)\right)-g^{*}\left(U^{i}\right)\right\|_{0}^{2} \\
\leq & \left(v^{i, H}-V^{i}, \beta^{-1}\left(v^{i, H}\right)-\beta^{-1}\left(V^{i}\right)\right)_{\Omega} \\
& +C \frac{H^{2}}{\delta^{2}}\left(\left\|\nabla v^{i, H}\right\|^{2}+\left\|\nabla V^{i}\right\|^{2}\right)+C \delta^{2 \mu} \\
\leq & \left(v^{i, H}-V^{i}, \beta^{-1}\left(v^{i, H}\right)-\beta^{-1}\left(V^{i}\right)\right)_{\Omega} \\
& +C\left(\frac{H^{2}}{\delta^{2}}+\delta^{2 \mu}\right), \\
\left\|\nabla g^{*}\left(u^{i, H}\right)\right\| & =\left\|\nabla g^{*}\left(I_{H} \beta_{\delta}^{-1}\left(v^{i, H}\right)\right)\right\|_{0} \leq C \frac{1}{\delta}\left\|\nabla v^{i, H}\right\|_{0} .
\end{aligned}
$$

So, we have

$$
\begin{aligned}
\left|T_{32}\right| \leq & \eta \tau \sum_{i=1}^{N}\left(v^{i, H}-V^{i}, \beta^{-1}\left(v^{i, H}\right)-\beta^{-1}\left(V^{i}\right)\right)_{\Omega} \\
& +C\left(\frac{H^{2}}{\delta^{2}}+\delta^{2 \mu}\right)+\tau \sum_{i=1}^{N}\left\|e_{u, i}^{H}\right\|_{-1}^{2} .
\end{aligned}
$$

Combining all the terms and choosing the parameter $\eta$ properly, we have

$$
\begin{aligned}
& \left\|e_{u, N}^{H}\right\|_{-1}^{2}+\sum_{i=1}^{N}\left\|e_{u, i}^{H}-e_{u, i-1}^{H}\right\|_{-1}^{2} \\
& \quad+\tau \sum_{i=1}^{N}\left(v^{i, H}-V^{i}, \beta^{-1}\left(v^{i, H}\right)-\beta^{-1}\left(V^{i}\right)\right)_{\Omega} \\
& \leq C\left(H^{2}+\frac{H}{\delta}+\frac{H^{2}}{\delta^{2}}+\delta^{\mu}+\delta^{2 \mu}+\frac{\epsilon^{2}}{l^{2}}\right) \\
& +\tau \sum_{i=1}^{N}\left\|e_{u, i}^{H}\right\|_{-1}^{2} .
\end{aligned}
$$

At last, we use the Gronwall inequality to the above formulation to finish the proof completely.

\section{Numerical Example}

In this subsection, we consider the Richards equation under the van Genuchten-Mualem model [2]. Consider

$$
\begin{gathered}
\partial_{t} \theta-\nabla \cdot\left(D(x, \theta) \nabla \theta+K(x, \theta) \cdot \vec{e}_{x_{3}}\right)=0 \\
\text { in }[0,1]^{2} \times(0, T), \\
\theta=\theta_{s} \quad \text { on } \Gamma_{t}=\left\{x_{1} \in(0,1), x_{3}=1\right\}, \\
\theta=\theta_{r} \quad \text { on } \Gamma_{b}=\left\{x_{1} \in(0,1), x_{3}=0\right\}, \\
\left(D(x, \theta) \nabla \theta+k(x, \theta) \vec{e}_{x_{3}}\right) \cdot \vec{n}=0 \\
\text { on } \Gamma_{l r}=\left\{x_{1}=0,1 ; x_{3} \in(0,1)\right\}, \\
\theta(x, 0)=\theta_{r} \quad \text { in }[0,1]^{2},
\end{gathered}
$$

and, here, the constitutive relations are

$$
\begin{aligned}
\theta(u) & =\theta_{r}+\left(\theta_{s}-\theta_{r}\right)\left(1+\alpha_{s}|u|^{n}\right)^{-m}, \\
K(x, \theta)= & K_{s}\left(x_{1}, x_{3}\right) \eta^{1 / 2}\left(1-\left(1-\eta^{1 / m}\right)^{m}\right)^{2}, \\
D(x, \theta)= & \frac{(1-m) K_{s}\left(x_{1}, x_{3}\right)}{\alpha_{s} m\left(\theta_{s}-\theta_{r}\right)} \eta^{(1 / 2)-m} \\
& \times\left(\left(1-\eta^{1 / m}\right)^{-m}+\left(1-\eta^{1 / m}\right)^{m}-2\right),
\end{aligned}
$$

where $\Theta=\left(\theta-\theta_{r}\right) /\left(\theta_{s}-\theta_{r}\right)$ and $K_{s}, \alpha_{s}, m$, and $n$ are the media parameters. Throughout this subsection, we set $m=0.5, n=$ $2, \theta_{r}=0.05$, and $\theta_{s}=0.489$. The heterogeneity comes from absolute permeability $K_{s}(x)$ and $\alpha(x)$.

Notice that the Richards equation under the van Genuchten-Mualem model (59) is degenerated at $\theta=\theta_{r}$ $(D=0)$ and $\theta=\theta_{s}(D=+\infty)$ and sharp interface would be developed between saturated and unsaturated regions. Our HMM-FEM scheme can be also used to treat this kind of problems.

In our computation, in order to avoid the difficulty of degeneration, the coefficient $D(x, \theta)$ is replaced by a regularized one as

$$
\bar{D}(x, \theta)= \begin{cases}D(x, \theta(\Theta)) & \Theta \in[0.001,0.999], \\ D(x, \theta(0.001)) & \Theta \in[0,0.001), \\ D(x, \theta(0.999)) & \Theta \in(0.999,1],\end{cases}
$$

where $\theta(\Theta)=\left(\theta_{s}-\theta_{r}\right) \Theta+\theta_{r}$

5.1. van Genuchten-Mualem Model with Periodic Coefficients. For periodic case, we set

$$
\begin{aligned}
& K_{s}\left(x_{1}, x_{3}\right)=\frac{1 /\left[2+1.8 \sin \left(2 \pi\left(2 x_{3}-x_{1}\right) / \epsilon\right)\right]}{117.4}, \\
& \alpha_{s}\left(x_{1}, x_{3}\right)=\frac{1 /\left[2+1.5 \sin \left(2 \pi\left(2 x_{3}-x_{1}\right) / \epsilon\right)\right]}{7.6} .
\end{aligned}
$$

In our simulation, $\epsilon$ is chosen to be $1 / 256$. 


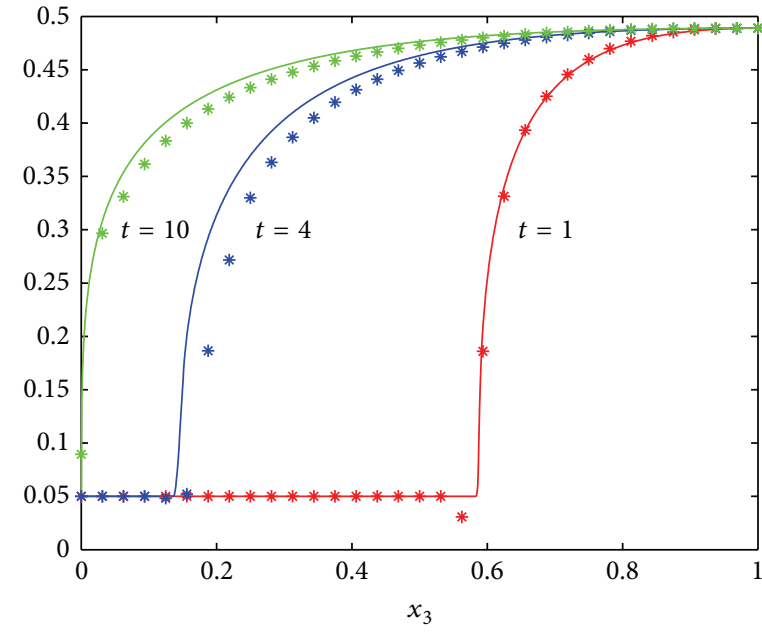

(a)

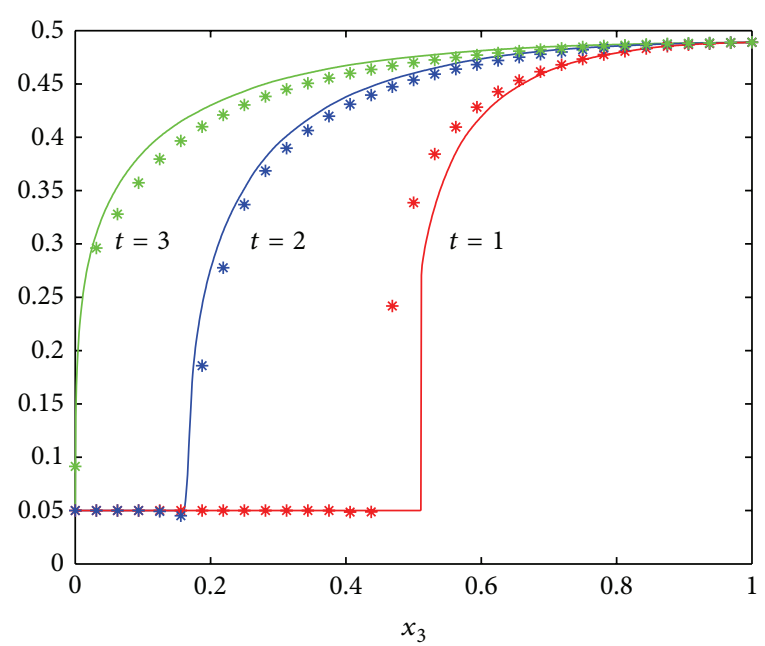

(b)

Figure 2: The HMM-FEM solution (star) compared with fine-scale solution (solid line) along the cross-section $x_{1}=0.5$ for periodic case (a) and random case (isotropic) (b), respectively.

The numerical solution of HMM-FEM is obtained on a macroscale grid $32 \times 32$. For each local sample cell $I_{l}$, we choose $l=4 \epsilon$ and solve the microproblems (25) on a grid with size of $\epsilon / 16$. We compare the HMM-FEM solution with the fine-scale solution which is obtained on a grid $4096 \times 4096$ by FVM. The time step is also chosen to be $\tau=1 / 1000$ in this subsection. We compare the HMM-FEM solution with the fine-scale solution along the cross-section of $x_{1}=0.5$ (Figure 2(a)).

5.2. van Genuchten-Mualem Model with Random Coefficients. For the random model, we only consider the isotropic heterogeneities case. We generate the random log-normal permeability fields $K_{s}(x)$ and $\alpha(x)$ by the same methods used in [16]. The corresponding normal distribution of $\log \left(K_{s}\right)$ is $N(-3,0.8)$ and the corresponding normal distribution of $\log \left(\alpha_{s}\right)$ is $N(-2,0.8)$. The correlation lengths of both $k_{s}$ and $\alpha_{s}$ are $l_{1}=l_{3}=0.01$ in $x_{1}$ and $x_{3}$ directions. The solution of HMM-FEM is obtained on a macroscale grid $32 \times 32$. For each local sample cell $I_{l}$, we choose $l=1 / 64$ and solve the microproblems (25) on a grid with size of $\delta / 16$. We compare the HMM-FEM solution with the fine-scale solution which is obtained by solving (59) on a grid $1024 \times 1024$ by FVM. We compare the HMM-FEM solution with the fine-scale solution along the cross-section $x_{1}=0.5$ (Figure 2(b)).

\section{Conflict of Interests}

The authors declare that there is no conflict of interests regarding the publication of this paper.

\section{Acknowledgments}

The research is supported by the Fundamental Research Funds for the Central Universities (no. 2013B10114), the Natural Science Foundation of Jiangxi Province (no. 20132BAB211018), and the NSF of China (no. 11271281).

\section{References}

[1] L. A. Richards, "Capillary conduction of liquids through porous mediums," Physics, vol. 1, pp. 318-333, 1931.

[2] M. Th. van Genuchten, "A closed-form equation for predicting the hydraulic conductivity of unsaturated soils," Soil Science Society of America Journal, vol. 44, no. 5, pp. 892-898, 1980.

[3] Y. Mualem, "A new model for predicting the hydraulic conductivity of unsaturated porous media," Water Resources Research, vol. 12 , no. 3, pp. 513-522, 1976.

[4] H. W. Alt and S. Luckhaus, "Quasilinear elliptic-parabolic differential equations," Mathematische Zeitschrift, vol. 183, no. 3, pp. 311-341, 1983.

[5] R. H. Nochetto, "Error estimates for multidimensional singular parabolic problems," Japan Journal of Applied Mathematics, vol. 4, no. 1, pp. 111-138, 1987.

[6] R. H. Nochetto and C. Verdi, "Approximation of degenerate parabolic problems using numerical integration," SIAM Journal on Numerical Analysis, vol. 25, no. 4, pp. 784-814, 1988.

[7] I. S. Pop, "Error estimates for a time discretization method for the Richards' equation," Computational Geosciences, vol. 6, no. 2, pp. 141-160, 2002.

[8] I. S. Pop and W. A. Yong, "A maximum principle based numerical approach to porous medium equation," in Proceedings of the 14th Conference on Scientific Computing, pp. 207-218, 1997.

[9] F. Radu, I. S. Pop, and P. Knabner, "Order of convergence estimate for an euler implicit mixed finite element discretization of Richards' equation," SIAM Journal on Numerical Analysis, vol. 4, pp. 1452-1478, 2004.

[10] W. Jäger and J. Kačur, "Solution of doubly nonlinear and degenerate parabolic problems by relaxation schemes," RAIRO Modélisation Mathématique et Analyse Numérique, vol. 29, no. 5, pp. 605-627, 1995.

[11] D. Kavetski, P. Binning, and S. W. Sloan, "Adaptive time stepping and error control in a mass conservative numerical solution 
of the mixed form of Richards equation," Advances in Water Resources, vol. 24, no. 6, pp. 595-605, 2001.

[12] I. S. Pop, F. A. Radu, and P. Knabner, "Mixed finite elements for the Richards' equation: linearization procedure," Journal of Computational and Applied Mathematics, vol. 168, pp. 13652373, 2004.

[13] M. Slodicka, "A robust and efficient linearization scheme for doubly nonlinear and degenerate parabolic paroblems arising in flow in porous medium," SIAM Journal on Scientific Computing, vol. 23, no. 5, pp. 1593-1614, 2002.

[14] T. Y. Hou and X.-H. Wu, "A multiscale finite element method for elliptic problems in composite materials and porous media," Journal of Computational Physics, vol. 134, no. 1, pp. 169-189, 1997.

[15] E. Weinan and B. Engquist, "The Heterogeneous multi-scale methods," Communications in Mathematical Sciences, vol. 1, pp. 87-132, 2003.

[16] Z. Chen, W. Deng, and H. Ye, "Upscaling of a class of nonlinear parabolic equations for the flow transport in heterogeneous porous media," Communications in Mathematical Sciences, vol. 3, no. 4, pp. 493-515, 2005.

[17] A. Abdulle, "The finite element heterogeneous multiscale method: a computational strategy for multiscale PDEs," in Multiple Scales Problems in Biomathematics, Mechanics, Physics and Numerics, vol. 31 of GAKUTO International Series Mathematical Sciences \& Applications, pp. 133-181, 2009.

[18] P. G. Ciarlet, The Finite Element Method for Elliptic Problems, North-Holland, Amsterdam, The Netherlands, 1978.

[19] H. T. Cao and X. Y. Yue, "Homogenization of Richards' equation of van Genuchten-Mualem model," Journal of Mathematical Analysis and Applications, vol. 412, no. 1, pp. 391-400, 2014. 


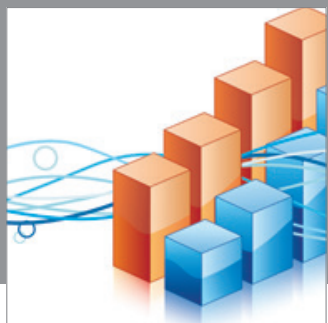

Advances in

Operations Research

mansans

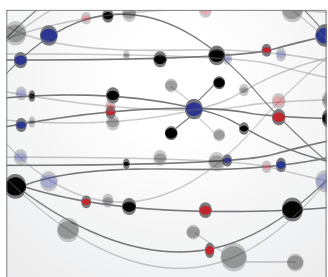

The Scientific World Journal
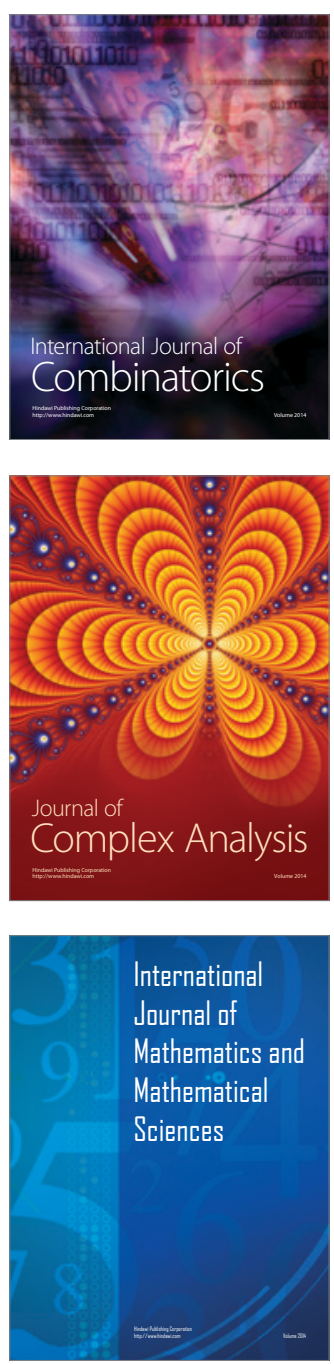
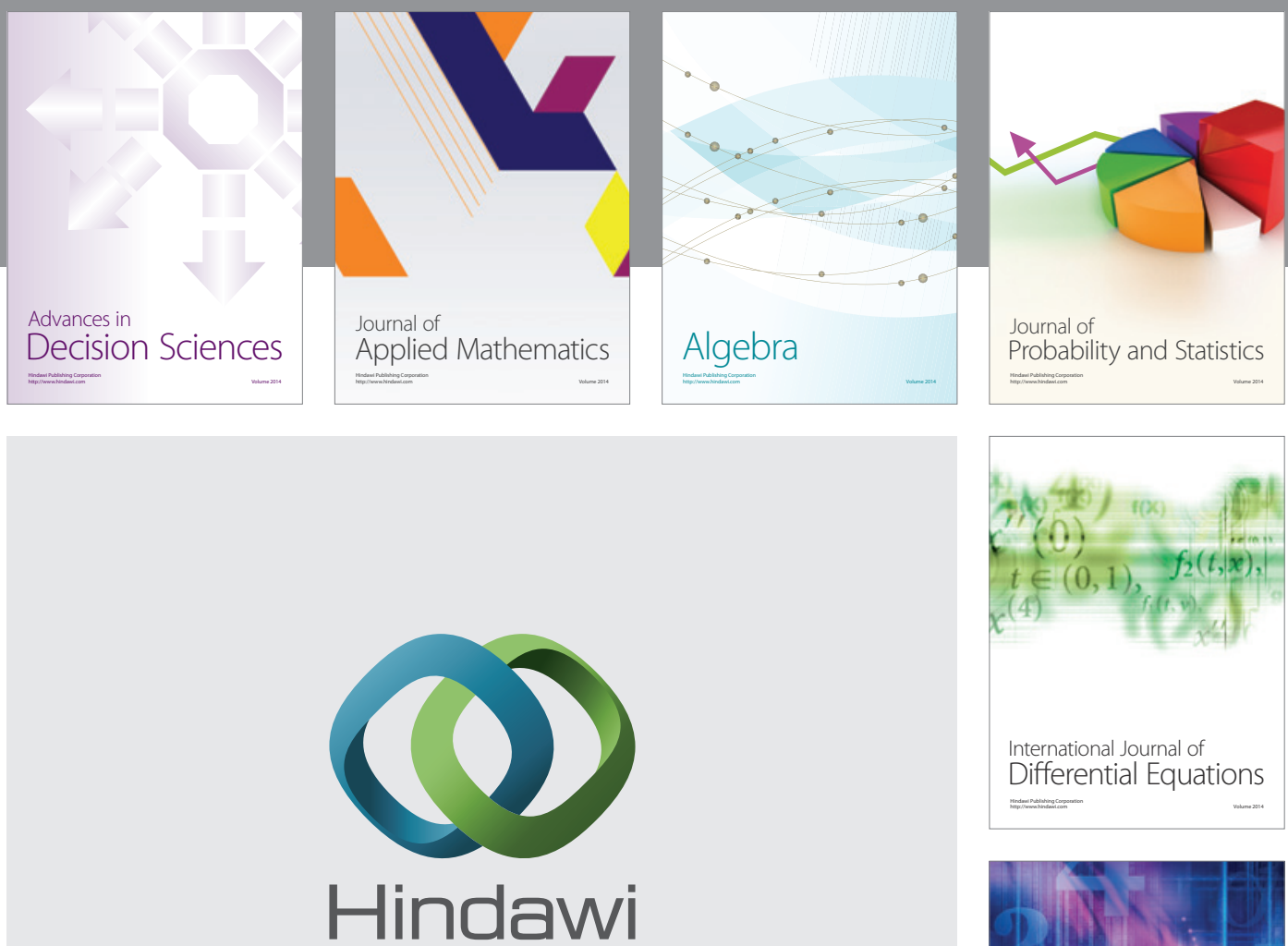

Submit your manuscripts at http://www.hindawi.com
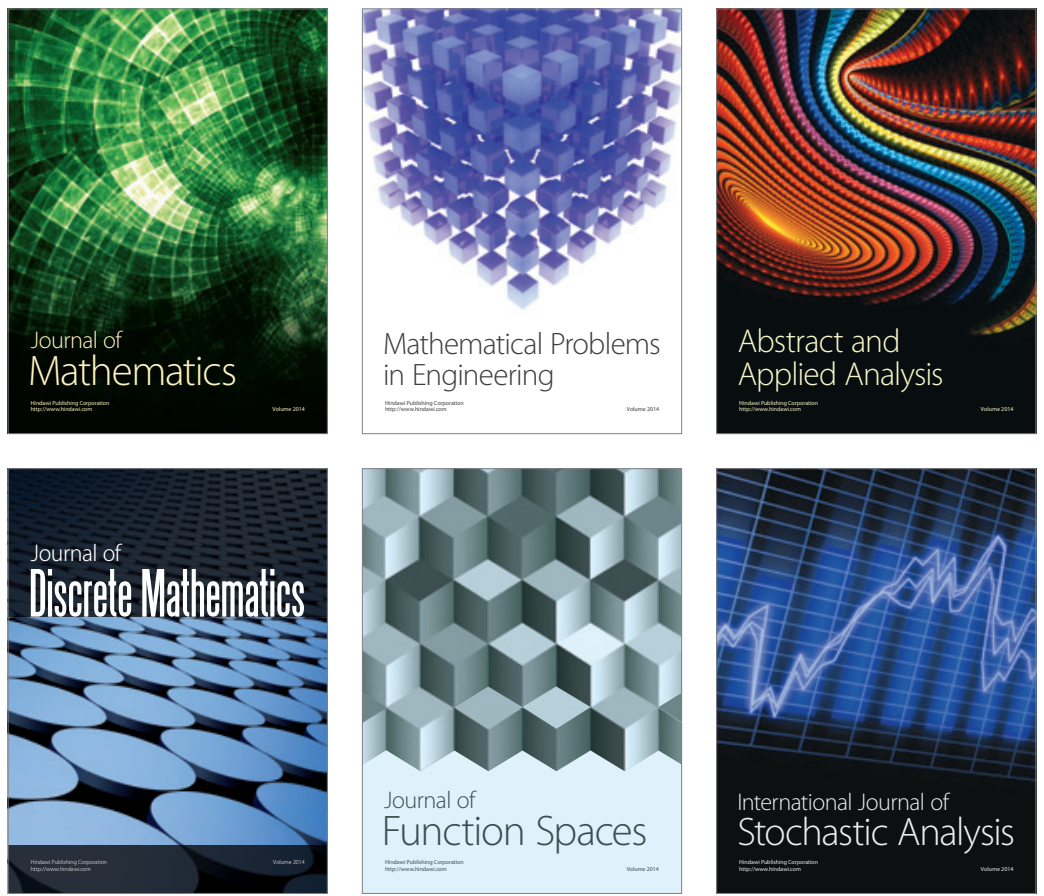

Journal of

Function Spaces

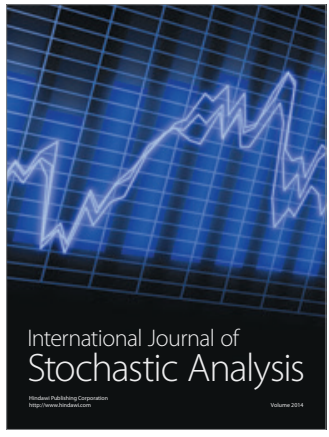

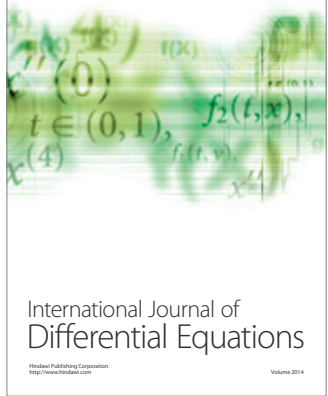
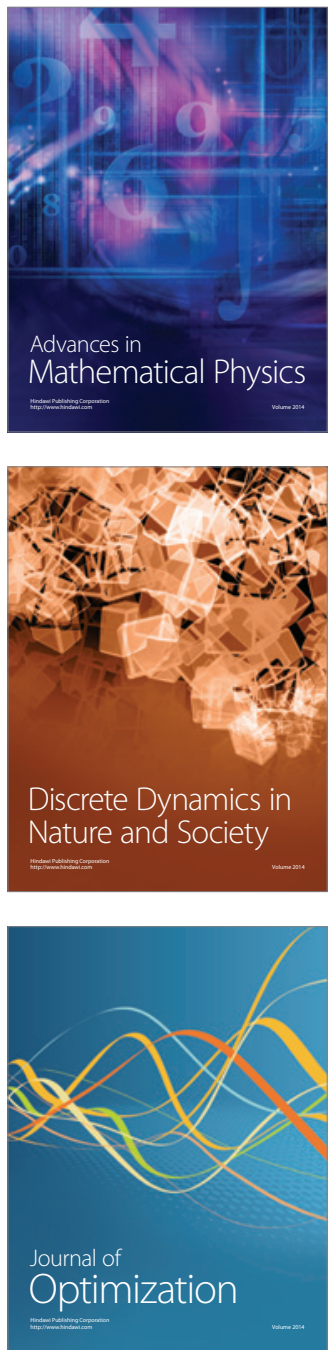